\title{
ANÁLISE BIBLIOMÉTRICA E SISTÊMICA SOBRE APLICAÇÃO DE MACHINE LEARNING PARA DIAGNÓSTICO DE FALHAS DE MÁQUINAS EM TEMPO REAL
}

Joelton Deonei Gotz (gotz@alunos.utfpr.edu.br) - Universidade Tecnológica Federal do Paraná (PPGEM-CT).

João Felipe Raffs Espolador (joaoe@alunos.utfpr.edu.br) - Universidade Tecnológica Federal do Paraná (PPGEM-CT).

Samuel Henrique Werlich (samuelw@alunos.utfpr.edu.br) - Universidade Tecnológica Federal do Paraná (PPGEM-CT).

Lucas Iuri dos Santos (lucassantos.1994@alunos.utfpr.edu.br) - Universidade Tecnológica Federal do Paraná (PPGEM-CT).

Milton Borsato (borsato@utfpr.edu.br) - Universidade Tecnológica Federal do Paraná (PPGEM-CT).

\section{RESUMO}

No processo de transformação digital, as fábricas do futuro buscam excelência com foco em qualidade de produto e eficiência de produção. Na busca de um processo controlado, objetivase reduzir ao máximo problemas e paradas em equipamentos, através da identificação de falhas de forma online, precoce e antecipada para realizar a tomada de decisão em tempo real. Nesse contexto, técnicas de Machine Learning (ML) são frequentemente utilizadas para diagnosticar falhas em equipamentos em tempo real. Portanto, esse trabalho busca recuperar as principais publicações vinculadas ao tema de diagnóstico de falhas em tempo real a partir da aplicação de técnicas de ML e realizar uma análise bibliométrica e sistêmica. Esse objetivo foi alcançado através da utilização do método de Knowledge Development Process-Constructivist (ProknowC) para selecionar e classificar os artigos mais relevantes ao tema. A aplicação do Proknow-C resultou no resgate de 38 artigos que compõem o portfólio final. Em seguida realizou-se uma análise bibliométrica que indicou a relevância dos artigos, as palavras-chaves mais encontradas e a tendência de publicações nessa área. Por fim, uma análise sistêmica foi efetuada, onde foram explorados os principais problemas observados, as tendências dos métodos mais utilizados e as oportunidades de pesquisa indicadas pelos autores.

Palavras chave: machine learning; diagnóstico de falhas; real time; deep learning 


\section{INTRODUÇÃO}

O advento da quarta revolução industrial está aliado à adoção da tecnologia da informação e a comunicação nos processos industriais. Com o objetivo de realizar o monitoramento e controle da qualidade dos produtos e da eficiência nos processos. Esse cenário origina o conceito "fábricas do futuro" ou ainda "fábricas inteligentes", as quais são caracterizadas por buscarem excelência em seus processos produtivos, além de se tornarem mais digitais, automatizadas e inteligentes (XU et al., 2019).

Na etapa intermediária do desenvolvimento de produto, encontra-se o Middle Of Life (MOL), que compreende a etapa mais longa da vida de um produto (JUN; KIRITSIS, 2012). Justamente na busca do prolongamento dessa etapa que está o segredo das fábricas do futuro: na qual buscam a adoção de sistemas de diagnóstico de falhas com abordagem preditiva. Nesse contexto, as falhas podem ser monitoradas nas fases iniciais, reduzindo-se gastos com manutenção corretiva e preventiva (WEN et al., 2018; XIA et al., 2020).

Atualmente são encontrados 3 modelos de aplicação de diagnóstico de falhas na indústria, dos quais o modelo baseado em Data-Driven é considerado muito promissor. Esse modelo trabalha com dados históricos que podem ser gerados a partir da digitalização das fábricas do futuro e realizar o reconhecimento de falhas a partir da análise do padrão de sinais (WEN et al., 2018).

Técnicas de ML são consideradas para aplicação de diagnóstico de falhas a partir de data-driven. Porém, os modelos estatísticos possuem dificuldade em extrair features de forma automática e de diferentes níveis de abstração. Além disso, precisam de grande conhecimento do processo para elaborar o modelo (HOANG; KANG, 2019). Para resolver esse problema, técnicas de ML baseadas em redes neurais podem ser utilizadas, principalmente pela sua capacidade de extrair features mais complexas automaticamente (EREN; INCE; KIRANYAZ, 2019).

Por isso, esse trabalho realizou uma revisão bibliográfica acerca do envolvimento de algoritmos de ML para diagnosticar falhas em equipamentos em tempo real através do Proknow-C. Esse processo auxilia o pesquisador a encontrar direções e trabalhos na área de pesquisa, com objetivo de verificar na academia as soluções publicadas recentemente, as dificuldades e as oportunidades de pesquisa encontradas nessa área (ENSSLIN et al., 2010). 
O método Proknow-C é composto das etapas de levantamento de artigos, análise bibliométrica e análise sistêmica, na qual é possível obter uma determinada quantidade de artigos que mostrarão as oportunidades de pesquisa na área visada (ENSSLIN et al., 2010). Por isso, esse artigo é composto por 5 seções: introdução, levantamento do portfólio bibliográfico, análise bibliométrica, análise sistêmica e a conclusão.

\section{LEVANTAMENTO DO PORTFÓLIO BIBLIOGRÁFICO}

A primeira etapa do método Proknow-C diz respeito ao levantamento do portfólio bibliográfico, que consiste na busca de artigos vinculados ao tema abordado. Para isso, criouse a sintaxe de pesquisa baseada no seguinte objetivo de trabalho: desenvolver um algoritmo de Machine Learning para diagnosticar falhas em tempo real em máquinas.

O objetivo foi dividido em três eixos, compostos das seguintes palavras-chaves: "Machine Learning", "diagnosticar" e "tempo real”. No entanto, para elaborar a sintaxe, palavras similares foram levantadas a ponto de construir uma sintaxe de busca otimizada, como visto no Quadro 1.

\begin{tabular}{|c||c||c|}
\hline \multicolumn{1}{|c||}{ Machine Learning } & diagnosticar & real time \\
\hline "artificial intelligence" & phm & "time series analy*" \\
\hline "deep learning" & "prognostic and health management" & "data-driven" \\
\hline "neural network*" & "failure analys*" & "online" \\
\hline "Long short-term memory" & "fault detection" & real-time data \\
\hline "Kalman Filter*" & "Fault diagnos*" & iot \\
\hline "random forest*" & "condition monitoring" & "edge computing" \\
\hline "feature learning" & "Predictive maintenance" & time-scale \\
\hline "genetic algorithm" & "predictive analys*" & temporal data \\
\hline "Bayesian Network" & "anomaly detection" & big data \\
\hline CNN or "convolutional neural network" & "sensor fault" & "signal processing" \\
\hline "extreme learning" & "fault identification" & time series \\
\hline Recurrent neural network & & data stream \\
\hline
\end{tabular}

QUADRO 1 - Palavras-chaves escolhidas para a montagem da sintaxe. Fonte: Os autores.

Após a montagem da sintaxe de pesquisa, foi realizado a busca nas quatro principais bases de artigos para engenharia: Compendex, Scopus, Web of Science e IEEE Xplorer. Para isso, a pesquisa foi direcionada a artigos periódicos e a publicações dos últimos cinco anos, restritos à língua inglesa. Também houve filtros de artigos vinculados com as áreas de Engenharia, Ciência da Computação e Energia. 
Com os filtros realizados e a sintaxe adequada para cada base de artigos, a pesquisa realizada entre os dias 02 e 27 de abril de 2021 retornou 2711 artigos como observado na Figura 1.

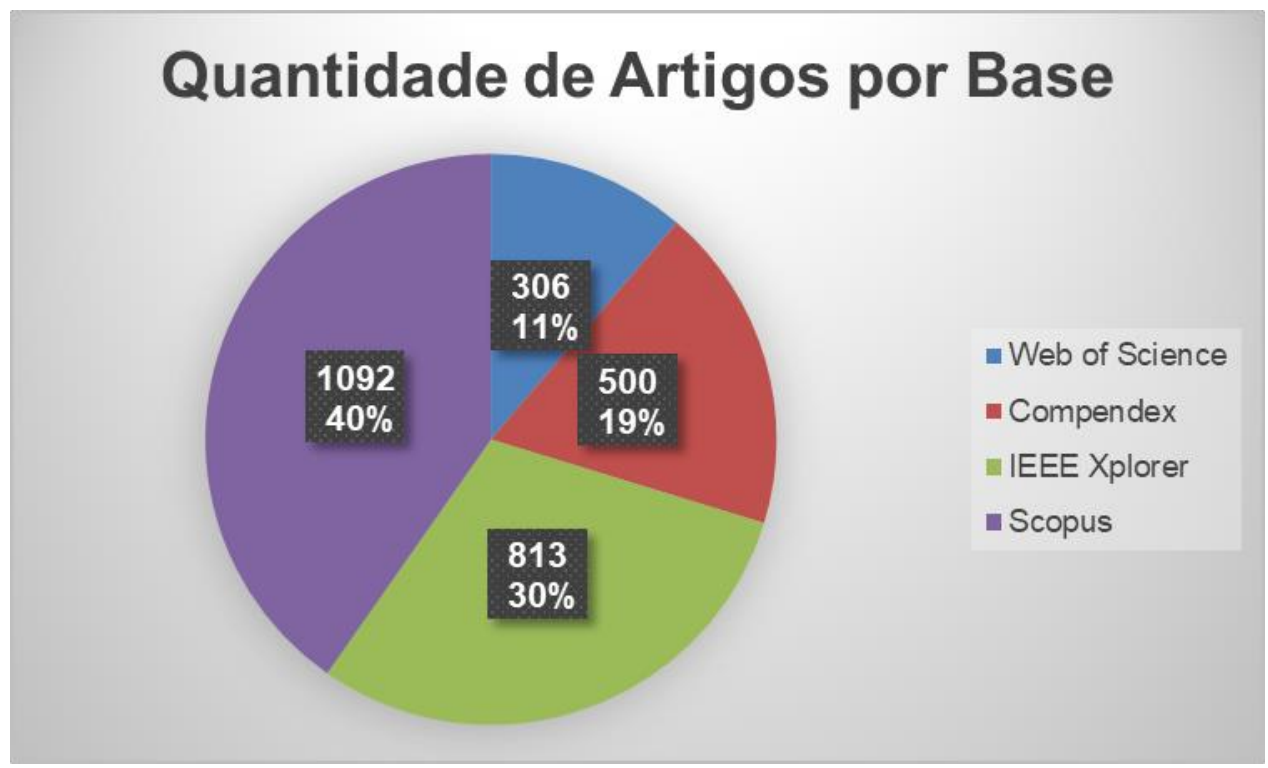

FIGURA 1 - Publicações por base de artigos. Fonte: Os autores.

A eliminação de artigos foi realizada através de um Script em linguagem Python. Ao final obteve-se 1947 artigos não repetidos em um único arquivo Excel. Essa etapa reduziu a quantidade de 2711 para 1947 artigos.

Em seguida foi realizada a leitura dos títulos dos artigos, que resultou em 1138 artigos relacionados ao tema. Após, foi aplicada a regra de Pareto para selecionar os $20 \%$ dos artigos que contém $80 \%$ do número total de citações, resultando em 265 artigos. Na etapa de repescagem dos $80 \%$ de artigos não escolhidos foram selecionados os artigos publicados nos dois últimos anos e os artigos escritos pelos autores mais relevantes, resultando em 711 artigos.

A seguir, foi realizada a leitura dos resumos dos 976 trabalhos, adotando o critério de seleção contido no Quadro 2. Após essa etapa, apenas 165 artigos alinhados com o tema e com um número relevante de citações foram mantidos.

\begin{tabular}{|c|c|}
\hline Critério & Número de Artigos \\
\hline Alinhados com o tema e relevante número de citações & 165 \\
\hline Desalinhados com o tema & 433 \\
\hline Alinhados com o tema, porém não descrevem detecção em tempo real & 14 \\
\hline Parcialmente alinhados com o tema, mas possui baixo número de citações & 364 \\
\hline
\end{tabular}

QUADRO 2 - Critérios de classificação na etapa de leitura dos resumos. Fonte: Os autores. 
Como o método Proknow-C recomenda que a leitura na integra dos artigos não seja realizada em número superior a 50 artigos, foi aplicado novamente o método de Pareto para selecionar $30 \%$ dos artigos com $70 \%$ das citações. Foram então obtidos 50 artigos que foram lidos na integra, sendo que 38 artigos foram considerados aptos ao tema proposto e estão apresentados no Quadro 3.

\begin{tabular}{|c|c|c|c|c|}
\hline Índice & Título & Ano & Citações & Autores \\
\hline 883 & A New Convolutional Neural Network-Based Data-Driven Fault Diagnosis Metho & 2018 & 516 & Long Wen , Xinyu Li , Liang Gao, Yu \\
\hline 1521 & Multisensor Feature Fusion for Bearing Fault Diagnosis Using Sparse Autoencod & 2017 & 304 & Zhuyun Chen, Weihua Li \\
\hline 1291 & Machine health monitoring using local feature-based gated recurrent unit netwo & 2017 & 255 & Rui Zhao, Dongzhe Wang, Ruqiang \\
\hline 702 & Multiscale Convolutional Neural Networks for Fault Diagnosis of Wind Turbine $\mathrm{C}$ & 2019 & 181 & Guoqian Jiang, Haibo He, Jun Yan, $\mathrm{P}$ \\
\hline 1035 & A convolutional neural network for fault classification and diagnosis in semicon & 2017 & 168 & Ki Bum Lee, Sejune Cheon, Chang O \\
\hline 899 & Deep Residual Networks with Dynamically Weighted Wavelet Coefficients for F & 2018 & 131 & Minghang Zhao, Myeongsu Kang, B \\
\hline 757 & Rolling element bearing fault diagnosis using convolutional neural network and & 2019 & 123 & Duy-Tang Hoang, Hee-Jun Kang \\
\hline 1066 & Transfer Learning with Neural Networks for Bearing Fault Diagnosis in Changing & 2017 & 122 & RAN ZHANG, HONGYANG TAO, LIFE \\
\hline 912 & A Data-Driven Design for Fault Detection of Wind Turbines Using Random Fores & 2018 & 119 & DAHAI ZHANG, LIYANG QIAN, BAIJII \\
\hline 1286 & A robust intelligent fault diagnosis method for rolling element bearings based o & 2018 & 110 & Xiang Li, Wei Zhang, Qian Ding \\
\hline 706 & \begin{tabular}{|l|} 
Fault diagnosis of wind turbine based on Long Short-term memory networks \\
\end{tabular} & 2019 & 107 & Jinhao Lei, Chao Liu, Dongxiang Jiar \\
\hline 914 & Convolutional neural network-based hidden Markov models for rolling element & 2018 & 105 & Shuhui Wang, Jiawei Xiang, Yongter \\
\hline 738 & A Generic Intelligent Bearing Fault Diagnosis System Using Compact Adaptive 1 & 2019 & 97 & Levent Eren, Turker Ince, Serkan Kir \\
\hline 635 & Fault diagnosis of gearbox based on RBF-PF and particle swarm optimization wa & 2019 & 87 & Liu Yang, Hanxin Chen \\
\hline 758 & A novel deeper one-dimensional CNN with residual learning for fault diagnosis & 2019 & 73 & DANDAN PENG, ZHILIANG LIU, HUA \\
\hline 1079 & \begin{tabular}{|l|} 
Bearing fault detection by one-dimensional convolutional neural networks \\
\end{tabular} & 2017 & 66 & Levent Eren \\
\hline 872 & Adaptive deep feature learning network with Nesterov momentum and its applic & 2018 & 46 & Shenghao Tanga, Changqing Shena, \\
\hline 1253 & Performance analysis of loT-based sensor; big data processing; and machine le & 2018 & 62 & Muhammad Syafrudin, Ganjar Alfiar \\
\hline 755 & A Digital-Twin-Assisted Fault Diagnosis Using Deep Transfer Learning & 2019 & 46 & YAN XU, YANMING SUN, XIAOLONG \\
\hline 703 & Automated bearing fault diagnosis scheme using $2 \mathrm{D}$ representation of wavelet & 2019 & 42 & M.M. Manjurul Islam, Jong-Myon Ki \\
\hline 884 & Lired: A light-weight real-time fault detection system for edge computing using & 2018 & 42 & Donghyun Park, Seulgi Kim, Yelin Ar \\
\hline 885 & Bayesian inference for mining semiconductor manufacturing big data for yield e & 2018 & 40 & Marzieh Khakifirooz, Chen Fu Chien, \\
\hline 578 & Data-Driven Safety Envelope of Lithium-Ion Batteries for Electric Vehicles & 2019 & 35 & Wei Li, Juner Zhu,Yong Xia,Maysam \\
\hline 814 & A Neural-Network-Based Method for RUL Prediction and SOH Monitoring of Lithi & 2019 & 26 & JIANTAO QU, FENG LIU, YUXIANG M \\
\hline 607 & Online Fault Diagnosis for Industrial Processes with Bayesian Network-Based Pr & 2019 & 19 & Wanke Yu, Chunhui Zhao \\
\hline 388 & Bayesian approach and time series dimensionality reduction to LSTM-based mo & 2020 & 18 & Diego Cabrera, Adriana Guamán, Sh \\
\hline 320 & Transformer fault diagnosis method using loT based monitoring system and ens & 2020 & 18 & Chaolong Zhang, Yigang He, Bolun \\
\hline 816 & An integrated imputation-prediction scheme for prognostics of battery data with & 2019 & 15 & Roozbeh Razavi-Far, Shiladitya Chak \\
\hline 613 & Remaining Useful Life Prediction of Lithium-Ion Batteries Using Support Vector h & 2019 & 11 & Yingzhou Wang, Yulong Ni, Shuai Lu \\
\hline 1496 & Single- and Multi-Fault Diagnosis Using Machine Learning for Variable Frequend & 2020 & 8 & Mohammad Zawad Ali, Md Nasmus \\
\hline 97 & Real-time robust forecasting-aided state estimation of power system based on & 2021 & 6 & Xingquan Ji, Ziyang Yin, Yumin Zhan \\
\hline 1422 & Remaining Useful Life Prognosis Based on Ensemble Long Short-Term Memory $\mathrm{N}$ & 2021 & 4 & Yiwei Cheng, Jun Wu, Haiping Zhu, \\
\hline 1380 & Data-driven prognosis method using hybrid deep recurrent neural network & 2020 & 4 & Min Xia, Xi Zheng, Muhammad Imra \\
\hline 285 & A coupling diagnosis method of sensors faults in gas turbine control system & 2020 & 2 & Rongzhuo Sun, Licheng Shi, Xilian Ya \\
\hline 347 & A tool wear monitoring and prediction system based on multiscale deep learnin & 2020 & 1 & Huihui Qiao, Taiyong Wang, Peng V \\
\hline 86 & Advanced fault diagnosis method for nuclear power plant based on convolutione & 2021 & 1 & Hang Wang, Min-jun Peng, Abiodun \\
\hline 35 & A Neural Network Based Method for Thermal Fault Detection in Lithium-Ion Batt & 2021 & 1 & Olaoluwa Ojo, Haoxiang Lang, Youn \\
\hline 1181 & SVM-BiLSTM: A Fault Detection Method for the Gas Station loT System Based on & 2020 & 0 & YAO JIAHAO, XIAONING JIANG, SHO \\
\hline
\end{tabular}

QUADRO 3 - Portfólio final. Fonte: Os autores.

\section{ANÁLISE BIBLIOMÉTRICA}

Após a formação do repositório final de artigos, foi realizada uma análise bibliométrica para exibir a relevância da base de artigos finais em função de uma ordem quantitativa (ENSSLIN et al., 2010). 
Dentre as análises realizadas, a Figura 2 mostra a quantidade de artigos publicadas por ano. Nela, é possível observar o ano de 2019 como destaque. Muito disso, se deve ao fato de que o tema está ganhando cada vez mais espaço, e os artigos de 2020 e 2021, por serem recentes, ainda estão com pouca relevância de citações.

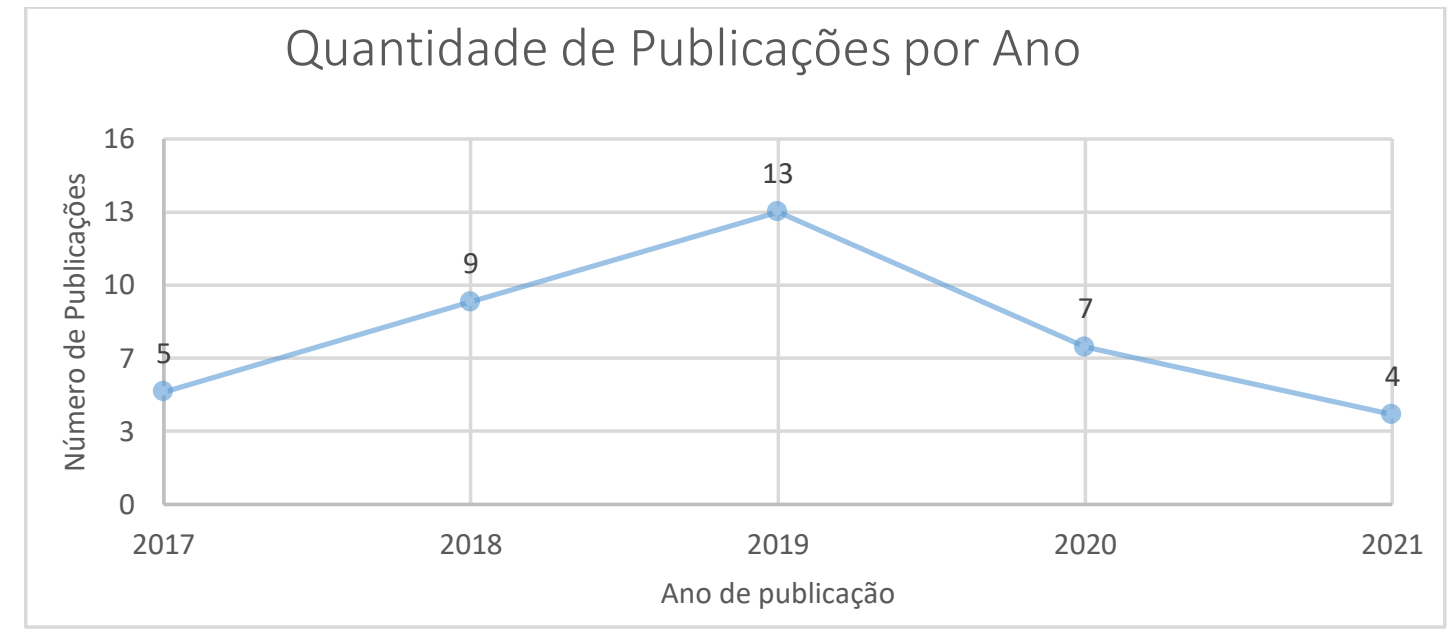

FIGURA 2 - Número de publicações por ano. Fonte: Os autores.

A Figura 3 mostra a relevância quanto ao número de citações, na qual pode-se destacar o artigo (WEN et al., 2018) com 516 citações e (CHEN; LI, 2017) com 304 citações.

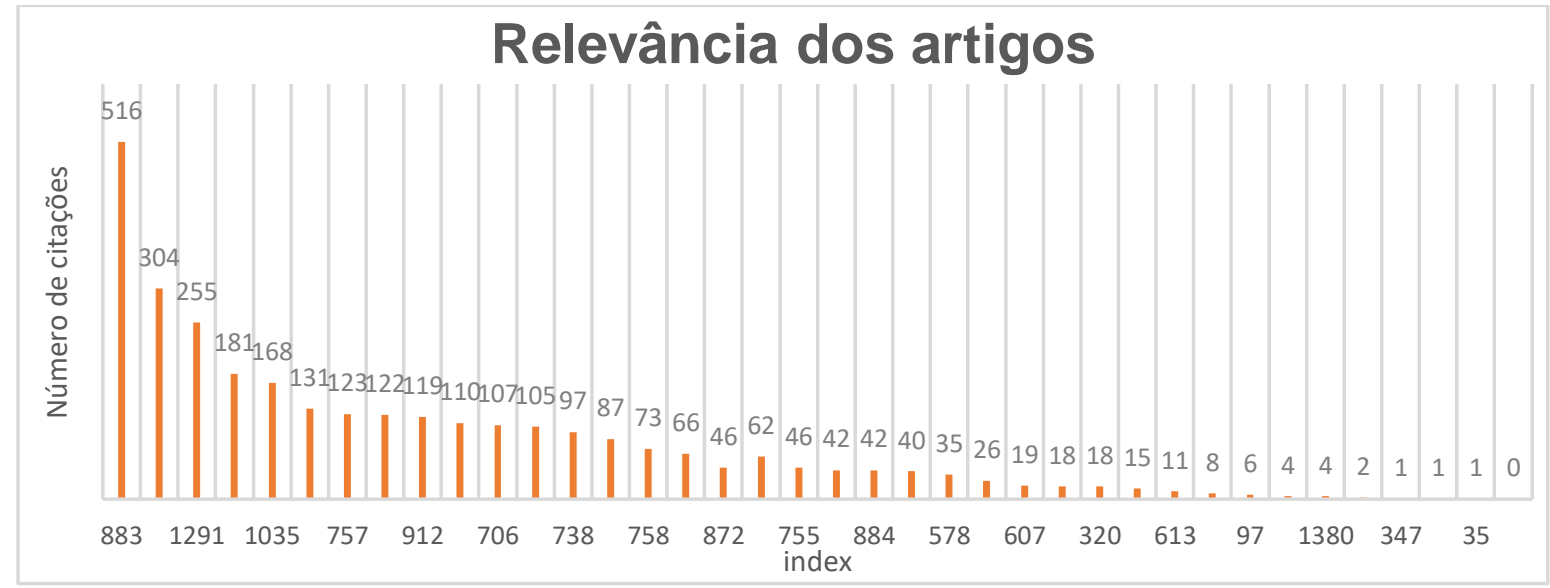

FIGURA 3 - Número de citações por artigos. Fonte: Os autores.

As palavras-chaves mais encontradas podem ser visualizadas na Figura 4. Destaque para "fault diagnosis", “convolution" e “deep learning” com 18, 11 e 10 publicações, respectivamente. 


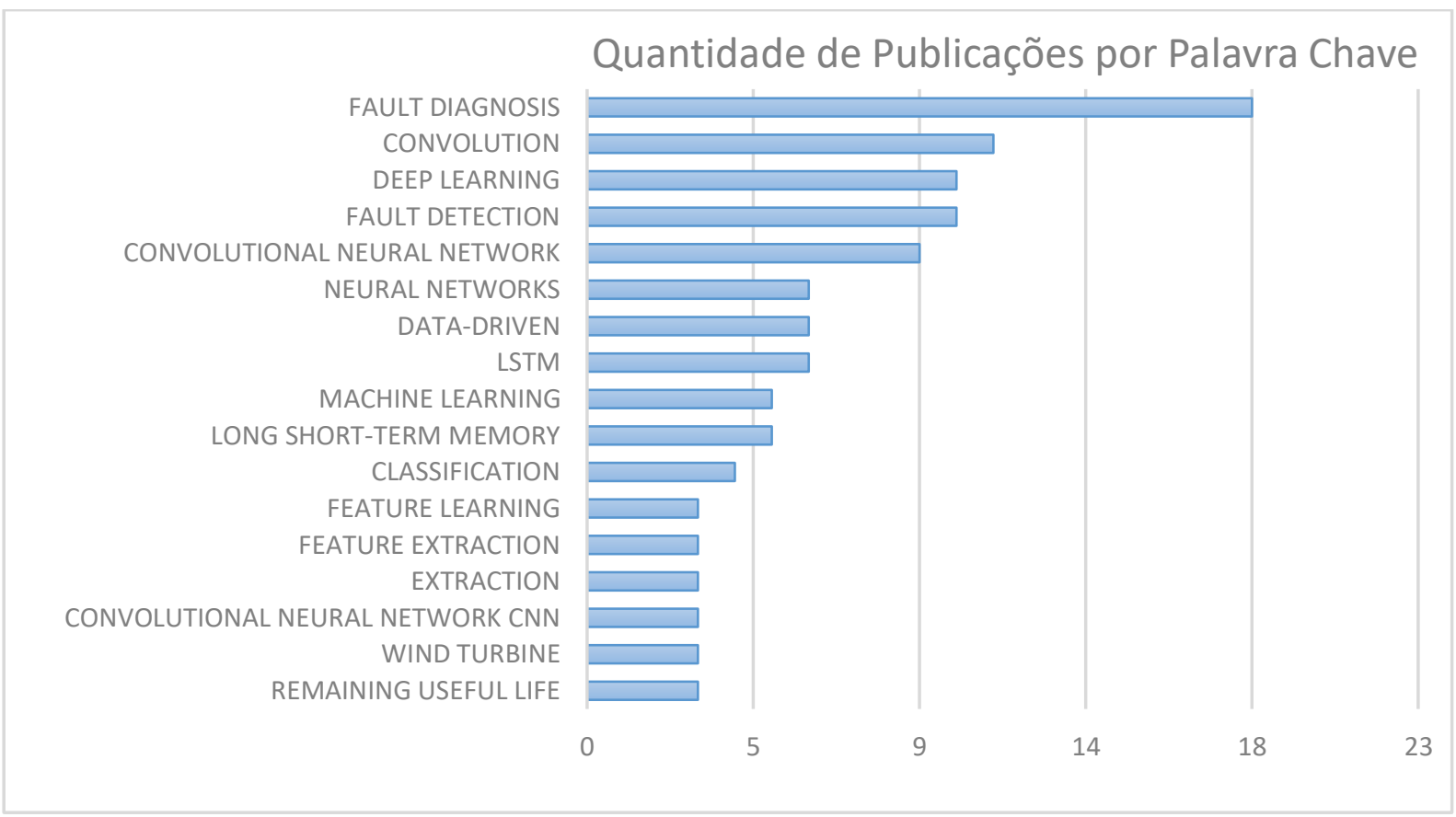

FIGURA 4 - Número de publicações por palavra-chave. Fonte: Os autores.

\section{ANÁLISE SISTÊMICA}

A partir da leitura dos 38 artigos que compõem o repositório final, foi realizada a análise sistemática. Para isso, foram extraídas as seguintes métricas dos artigos finais: problemas, soluções e oportunidades, as quais serão descritas a seguir.

\subsection{Análise do Problema}

Foram identificados cinco grupos de problemas, que serão mencionados a seguir:

4.1.1 Processo de digitalização: o advento da quarta revolução industrial trouxe à tona o conceito de fábricas inteligentes, as quais buscam excelência de controle de qualidade dos produtos e eficiência de processos que podem ser alcançados devido ao emprego de automação (XU et al., 2019; YU; ZHAO, 2019). Park et al. (2018) indicam que o segredo das fábricas do futuro está relacionado com o diagnóstico precoce de falhas atra vés do monitoramento da vida útil de equipamento. Isso leva, segundo Xia et al. (2020), à redução de paradas de produção baseadas nas manutenções preventivas e na diminuição de custos com substituições de elementos devido a manutenções corretivas.

4.1.2 Dificuldade de extração de features: um dos problemas encontrados nos trabalhos pesquisados está na extração de features, demonstrado no Quadro 4. Wen et al. (2018) indicam que nunca foi visto tanta disponibilidade de dados como nos dias de hoje, porém modelos de 
ML que trabalham de forma estatística se tornaram ineficientes com o crescimento da geração de dados e o incremento de complexidade dos equipamentos industriais (HOANG; KANG, 2019).

\begin{tabular}{|l|l|}
\hline \multicolumn{1}{|c|}{ Trabalhos } & \multicolumn{1}{c|}{ Descrição da abordagem } \\
\hline (CHEN; LI, 2017) & $\begin{array}{l}\text { Apontam o processo árduo e exaustivo de extração manual de features, a qual também é totalmente dependente } \\
\text { de conhecimentos prévios de operadores para realizar o processamento dos dados. }\end{array}$ \\
\hline $\begin{array}{l}\text { (ZHANG et al. , 2017) e (XU } \\
\text { et al. , 2019) }\end{array}$ & $\begin{array}{l}\text { Relatam a dificuldade desses modelos atuarem em condições de operações de máquinas, nas quais não foram } \\
\text { treinados, ou na classificação de falhas não vistas no treinamento. }\end{array}$ \\
\hline (LI; ZHANG; DING, 2018) & $\begin{array}{l}\text { Acrescentam que as máquinas estão em plena mudança dos parâmetros de operação, logo o treinamento que é } \\
\text { realizado para certas distribuições de dados não atende uma máquina por muito tempo }\end{array}$ \\
\hline (ZHAO et al. , 2018) & $\begin{array}{l}\text { Citam que as técnicas de ML estatística tem dificuldade de trabalhar com dados em tempo real por não } \\
\text { apresentarem habilidade para extrair features em dados de alta frequência, normalmente encontrados em sinais } \\
\text { de vibração e corrente. }\end{array}$ \\
\hline
\end{tabular}

QUADRO 4 - Trabalhos que demonstram a dificuldade de extrair features. Fonte: Os autores.

Yu e Zhao (2019) apontam para a realidade de existirem múltiplas falhas na indústria. Consequentemente, Ali et al. (2020) relatam a dificuldade de encontrar algoritmos que realizam diagnostico de múltiplas falhas. Cheng et al. (2021) indicam que utilizar um único algoritmo para detectar falhas reduz a eficiência do diagnóstico, pois nem todas as falhas podem ser facilmente identificadas.

4.1.3 Problemas nos sinais: Ojo et al. (2021) citam que falhas em sensores geram problemas críticos para modelos de ML, pois podem causar predições equivocadas. Segundo Sun et al. (2020), o crescimento da complexidade de sistemas de controle na indústria resulta no aumento do número de sensores, que reflete no incremento de falhas. Por isso, antes mesmo de realizar o diagnóstico de falhas, é necessário detectar falhas em sensores. Alguns trabalhos que demonstraram a preocupação com os ruídos podem ser vistos no Quadro 5.

\begin{tabular}{|l|l|}
\hline \multicolumn{1}{|c|}{ Trabalhos } & \multicolumn{1}{c|}{ Descrição da abordagem } \\
\hline (SYAFRUDIN et al ., 2018) & Indicam que a presença de ruídos e outliers nos dados, prejudica a acurácia dos modelos de classificação \\
\hline (HOANG; KANG, 2019) & $\begin{array}{l}\text { Mencionam que sinais provenientes de máquinas possuem ruídos, por isso é necessário métodos para a } \\
\text { limpeza dos dados }\end{array}$ \\
\hline (PENG et al. , 2019) & $\begin{array}{l}\text { Abordam a existência de ruídos em sinais de vibração de rolamentos de rodas de trem, principalmente ruídos } \\
\text { de baixa frequência }\end{array}$ \\
\hline (LI; ZHANG; DING, 2018) & Relatam a presença de ruídos em dados de rolamentos. \\
\hline
\end{tabular}

QUADRO 5 - Trabalhos que demonstram a presença de ruídos nos dados. Fonte: Os autores.

4.1.4 Arquitetura em nuvem: Park et al. (2018) indicam o crescente uso de sistemas em nuvem para acelerar a aplicação de soluções de diagnóstico de falhas, principalmente na análise de extensas quantidades de dados. Porém, Qiao, Wang e Wang (2020) apontam a presença de 
latência na entrega de pacotes para diagnósticos em tempo real. Além disso, o custo de soluções em nuvem está diretamente atrelado ao armazenamento e processamento de dados.

4.1.5 Motivações extras: Esse trabalho levantou processos considerados críticos devido a sua complexidade, que necessitam de sistemas de diagnóstico de falhas e serão apresentados no Quadro 6.

\begin{tabular}{|c|c|}
\hline Casos de uso & Descrição do trabalho \\
\hline \multirow{2}{*}{$\begin{array}{l}\text { Monitoramento de } \\
\text { baterias de íons-lítios }\end{array}$} & $\begin{array}{l}\text { Qu et al. (2019) indica o crescente uso de baterias de íons-lítio em várias aplicações, principalmente em } \\
\text { mobilidade. }\end{array}$ \\
\hline & $\begin{array}{l}\text { Porém Wang et al. (2019) acena para a presença de sérios riscos relacionados a sua aplicabilidade, } \\
\text { principalmente pela presença de elementos químicos e físicos que possuem comportamentos atípicos em altos } \\
\text { níveis de degradação. }\end{array}$ \\
\hline Transformadores & $\begin{array}{l}\text { Elementos sensíveis a falhas, principalmente pela exposição ao ambiente, como sol e chuva (ZHANG et al. , } \\
\text { 2020). }\end{array}$ \\
\hline \multirow[b]{2}{*}{ Turbinas eólicas } & Jiang et al. (2019) aponta para ocorrência de falhas de turbinas devido à exposição ao ambiente. \\
\hline & $\begin{array}{l}\text { Zhang et al. (2018) complementa que um dos motivos desses sistemas de geração de energia eólica ainda são } \\
\text { considerados caros, pois estão sujeitos a frequente ocorrência de falhas, principalmente nas caixas redutoras e } \\
\text { rolamentos. }\end{array}$ \\
\hline Usinas Nucleares & $\begin{array}{l}\text { Wang et al. (2021) descreve o a necessidade de um sistema de diagnóstico de falhas em usinas nucleares devido } \\
\text { ao risco de vazamento de elementos radioativos }\end{array}$ \\
\hline \multirow[b]{2}{*}{ Motores Elétricos } & Eren (2017) comenta sobre a importância de motores elétricos nas indústrias. \\
\hline & $\begin{array}{l}\text { Ali et al. (2020) complementa, que o correto funcionamento e o prolongamento da vida útil dependem } \\
\text { exclusivamente de um bom sistema de diagnóstico de falhas que possa identificar de forma inicial o surgimento } \\
\text { de eventos que que levam a possíveis falhas e inclusive danificação completa o dispositivo. }\end{array}$ \\
\hline Rolamentos & $\begin{array}{l}\text { Rolamentos são citados em vários trabalhos, principalmente pela alta criticidade em máquinas rotativas, pois são } \\
\text { os elementos que mais geram falhas (HOANG; KANG, EREN; INCE; KIRANYAZ, 2019) }\end{array}$ \\
\hline
\end{tabular}

QUADRO 6 - Aplicação de diagnóstico de falhas em casos de uso. Fonte: Os autores.

\subsection{Análise das Soluções}

As análises de tendências de soluções para o diagnóstico de falhas em tempo real foram agrupadas em 4 grupos:

4.2.1 Extração automática de features: devido às dificuldades encontradas pelos modelos de estatísticos de ML em detectar falhas, foi visto, nessa pesquisa, uma tendência para o uso de técnicas de DL (QIAO; WANG; WANG, 2020). Zhang et al. (2017) indicam que DL tem a característica de automatizar a extração de features. Wen et al. (2018) complementam que modelos de DL têm a capacidade de extrair features abstratas de dados brutos de forma automática. E, por fim, Chen e Li (2017), Syafrudin et al. (2018) e Xia et al. (2020) indicam ótima performance de DL para atuar na fusão de dados a partir de múltiplos sensores. Essa medida se torna importante pois a acurácia do diagnóstico de falhas pode ser incrementada com os dados se complementando.

4.2.2 Tendência de modelos: os modelos mais vistos para diagnóstico de falhas foram o Convolution Neural Network (CNN) e o Long Short-Term Memory (LSTM). CNN é uma rede 
neural que pode trabalhar no domínio do tempo em uma dimensão (1D) ou na conversão em imagens no domínio da frequência em duas dimensões (2D). Segundo Wen et al. (2018), esse modelo tem como característica fundamental a redução da dimensionalidade das features extraídas. Eren (2017) e Eren, Ince e Kiranyaz (2019) mencionam que, a partir do emprego do modelo 1D-CNN, é possível realizar a atuação em time-series sem a necessidade de préprocessamento dos dados. Já Jiang et al. (2019) indicam que o modelo 2D-CNN trabalha com a transformação dos dados para o domínio da frequência, convertendo os sinais em imagens, a partir das quais as features podem ser extraídas. Variações do modelo original são vistas no Quadro 7.

\begin{tabular}{|l|l|l|}
\hline \multicolumn{1}{|c|}{ Autor } & \multicolumn{1}{|c|}{ Derivação } & \multicolumn{1}{c|}{ Objetivo } \\
\hline (PENG et al. , 2019) & $\begin{array}{l}\text { 1D-CNN com residual learning e } \\
\text { convolutional kernel }\end{array}$ & Detectar falhas em ambientes ruidosos \\
\hline $\begin{array}{l}\text { (ZHAO et al. , 2018) \& } \\
\text { (ISLAM; KIM, 2019) }\end{array}$ & Wavelet & Transformar dados para domínio da frequência \\
\hline (WANG et al. , 2018) & Modelo de Markov com 2D-CNN & $\begin{array}{l}\text { Predição de probabilidades de estados futuros nas } \\
\text { funções de transição. }\end{array}$ \\
\hline
\end{tabular}

QUADRO 7 - Derivações de CNN. Fonte: Os autores.

Já redes LSTM têm a habilidade de indicar predições em tempo real, pois podem trabalhar com dados passados para refletir nas predições do futuro (CHENG et al., 2021). Ojo et al. (2021) complementam que o modelo realiza predições de curto prazo com memórias de longo prazo. Algumas abordagens relacionadas com LSTM estão no Quadro 8.

\begin{tabular}{|l|l|}
\hline \multicolumn{1}{|c|}{ Autor } & \multicolumn{1}{c|}{ Objetivo } \\
\hline (XIA et al ., 2020) & $\begin{array}{l}\text { LSTM para realizar a predição de Remaining Useful life de forma online e em tempo } \\
\text { para tomada de decisão. }\end{array}$ \\
\hline (CABRERA et al ., 2020) & $\begin{array}{l}\text { LSTM com otimizador dos parâmetros realizado pelo modelo Bayesian Optimization para } \\
\text { trabalhar no diagnóstico de falhas em tempo real de compressores }\end{array}$ \\
\hline (LEI; LIU; JIANG, 2019) & LSTM para diagnosticar falhas de múltiplos sensores com a fusão de dados \\
\hline
\end{tabular}

QUADRO 8 - Abordagens complementares de LSTM. Fonte: Os autores.

Como relatado por Cheng et al. (2021), o uso de um único classificador para processos complexos pode se tornar limitante. Por isso, entra em contexto o Ensemble Learning (EL), o qual utiliza vários classificadores para serem treinados e diagnosticar falhas, construindo, dessa maneira, uma variedade de hipóteses que podem ser observadas para resolver um determinado problema (YU; ZHAO, 2019). Alguns trabalhos sobre esse tema estão no Quadro 9. 


\begin{tabular}{|l|l|}
\hline \multicolumn{1}{|c|}{ Trabalhos } & \multicolumn{1}{c|}{ Descrição da abordagem } \\
\hline (SYAFRUDIN et al ., 2018) & Fusão de 4 algoritmos em um processo no setor automotivo \\
\hline (ZHANG et al. , 2020) & $\begin{array}{l}\text { Construção de um modelo de EL baseado em DBN e auto-encoder para } \\
\text { eliminação de ruídos e classificadores individuais para identificar falhas e suas } \\
\text { localizações em uma rede elétrica }\end{array}$ \\
\hline (ZHANG et al. , 2018) & $\begin{array}{l}\text { Uma combinação de Random Forest e Extreme Gradiente Boosting na detecção } \\
\text { de falhas de turbinas eólicas }\end{array}$ \\
\hline (JIAHAO et al. , 2020) & $\begin{array}{l}\text { Fusão de SVM com BiLSTM para diagnosticar falhas com alta acurácia em dados } \\
\text { provenientes de dispositivos IOT }\end{array}$ \\
\hline
\end{tabular}

QUADRO 9 - Descrição do uso de EL em trabalhos contidos no repositório Final. Fonte: Os autores.

Outra tendência observada na pesquisa está relacionada com a transferência de aprendizagem. Zhang et al. (2017) indicam que os problemas de diferença entre dados de treinamento e de teste podem ser resolvidos com algoritmos chamados de Transfer Learning (TF). O objetivo dessa técnica é realizar o treinamento sobre dados que possuem uma determinada característica e transferir o modelo para realizar a inferência em dados similares, porém com outras características. Uma das aplicações desse modelo foi vista no desenvolvimento de um Digital Twin. Esse modelo virtual é capaz de simular dados de operações da máquina sob diferentes condições de trabalhos, além de simular falhas que podem ser utilizadas para treinar o modelo (XU et al., 2019). Outras técnicas vistas na revisão estão presentes no Quadro 10.

\begin{tabular}{|c|l|}
\hline Técnicas & \multicolumn{1}{c|}{ Descrição } \\
\hline $\begin{array}{c}\text { Digital Twin, Convolution Gated Recurrent } \\
\text { unit e Particle Swarm Optimization }\end{array}$ & $\begin{array}{l}\text { Wang et al. (2021) desenvolveu um Digital Twin para simular dados de processo. Em seguida, aliou esses } \\
\text { dados do modelo virtual com as técnicas de Convolution Gated Recurrent Unit (CGRU) com otimização dos } \\
\text { hiperparâmetros e um método de inchamento de partículas para diagnosticar falhas em equipamentos de } \\
\text { usinas nucleares }\end{array}$ \\
\hline $\begin{array}{c}\text { Particle Swarm Optimization, attention } \\
\text { mechanism e LSTM }\end{array}$ & $\begin{array}{l}\text { Qu et al. (2019) utilizou o inchamento de partículas para otimizar os hiperparâmetros e aliou a um método } \\
\text { chamado attention mechanism para calcular os pesos das features, e complementou com LSTM para estimar } \\
\text { State of Health (SOH) e realizar predições de Remaining Useful Life (RUL) de baterias }\end{array}$ \\
\hline $\begin{array}{c}\text { Decomposition Wavelet Transformation } \\
\text { (DWT), Decision Tree Learning, Support } \\
\text { Vector Machine e K-Nearest Neighbor }\end{array}$ & $\begin{array}{l}\text { li et al. (2020) utilizou o DWT para transformar os dados no domínio da frequência, em várias faixas } \\
\text { diferentes que permitem analisar os sinais de com multi resoluções. Após a extração das features, foram } \\
\text { utilizados alguns classificadores como Decision Tree Learning, SVM e KNN e classificadores ensemble que uni } \\
\text { vários algoritmos para classificar as falhas }\end{array}$ \\
\hline $\begin{array}{c}\text { Detecção de Anomalias e density-based } \\
\text { spational clustering }\end{array}$ & $\begin{array}{l}\text { Syafrudin et al. (2018) aplicaou técnicas de deteç̧ão de outliers nos dados, entre elas está a density-based } \\
\text { spational clustering para trabalhar com dados que contém alto nível de ruídos }\end{array}$ \\
\hline $\begin{array}{c}\text { Wavelet e Support Vector Machine (SVR) } \\
\text { dividido em extração de features em sinais e classificação de features, onde o Wavelet pode extrair features } \\
\text { dos sinais e um classificador SVR é utilizado para diagnosticar e classificar as falhas nos sensores. Foram } \\
\text { utilizada as 5 principais falhas de sinais de sensores. }\end{array}$ \\
\hline
\end{tabular}

QUADRO 10 - Descrição de técnicas complementares observadas na pesquisa. Fonte: Os autores.

4.2.3 Computação em borda $x$ nuvem: Outra abordagem vista nas pesquisas está no local de armazenamento e processamento dos dados. Park et al. (2018) indicaram que o uso de nuvem permitiu o armazenamento e análise de dados em grande escala, sem a dependência de 
estruturas físicas locais que demandam enorme investimento de recursos e manutenção. Por outro lado, o autor menciona o uso da tecnologia de computação de borda para eliminar o problema de latência de transmissão. Computação de borda, também conhecida como Edge Computing é existência da aplicação de um processo de computação que se localiza entre a fonte de geração de dados e o armazenamento em nuvem (SHI et al., 2016). Para complementar, Qiao, Wang e Wang (2020) relatam para o uso de uma combinação entre computação em nuvem e em borda para reduzir os altos custos de estrutura em nuvem.

4.2.4 Prognostic and health management (PHM): outro assunto abordado na pesquisa foi o emprego de PHM. Park et al. (2018) mencionaram a importância do PHM na indústria para realizar prognósticos de falhas e predições do fim de vida útil de equipamento. Segundo os autores, a aplicação de PHM se torna mais eficiente com o uso do Data-Driven, que permite a coleta e armazenamento de uma grande quantidade de dados, o que é útil para a análise de padrão de dados. Xia et al. (2020) relatam ainda que o PHM ganha cada vez mais espaço no prognóstico de condições de operação dos equipamentos e no auxílio na gestão de manutenção.

\subsection{Análise das Oportunidades}

Abaixo serão listados três grupos com indicações de oportunidades.

4.3.1 Melhorias no método atual: a maioria dos trabalhos sugeriu melhorias que serão realizadas nos próprios modelos, como visto no Quadro 11.

\begin{tabular}{|c|c|}
\hline Trabalhos & Descrição das oportunidades \\
\hline (WEN et al. , 2018) & Indicam a necessidade de adaptação do atual modelo para diagnosticar falhas desconhecidas \\
\hline (LEE; CHEON; KIM, 2017) & Mencionam necessidade de aprofundamento da técnica utilizada \\
\hline $\begin{array}{l}\text { (ZHANG et al. , 2018), (EREN; INCE; } \\
\text { KIRANYAZ, 2019), (XIA et al. , 2020) e } \\
\text { (LEI; LIU; JIANG, 2019) }\end{array}$ & $\begin{array}{l}\text { Indicam a necessidade de mais dados e diferentes condições de trabalho para serem utilizadas e } \\
\text { testadas }\end{array}$ \\
\hline (LI; ZHANG; DING, 2018) & Apontam otimização da estrutura da rede como sugestão de melhoria. \\
\hline $\begin{array}{l}\text { (EREN, 2017) \& (SYAFRUDIN et al. , } \\
\text { 2018) }\end{array}$ & Acenam para que mais dados de falhas seja utilizados para o modelo \\
\hline (ISLAM; KIM, 2019) & Sugerem a aplicação do modelo em diagnóstico de falhas reais \\
\hline (PARK et al. , 2018) & $\begin{array}{l}\text { Apontam para a necessidade de dispositivo com mais memória, além requisitar a redução do } \\
\text { tamanho de dados para treinamento }\end{array}$ \\
\hline $\begin{array}{l}\text { (YU; ZHAO, 2019), (WANG et al. , 2021) } \\
\text { e (ZHANG et al. , 2020) }\end{array}$ & A validação deve ser realizada no diagnóstico de falhas outros sistemas \\
\hline (RAZAVI-FAR et al., 2019) & $\begin{array}{l}\text { Como sugestão, os autores indicam o uso de múltiplas saídas e técnicas de trabalho em paralelo } \\
\text { para reduzir o custo computacional }\end{array}$ \\
\hline $\begin{array}{l}\text { (Jl et al. , 2021), (JIAHAO et al. , 2020) e } \\
\text { (CHENG et al. , 2021) }\end{array}$ & $\begin{array}{l}\text { Os autores mencionam a necessidade de melhorias para diagnosticar falhas em diferentes } \\
\text { condições de trabalho e falhas desconhecidas }\end{array}$ \\
\hline (QIAO; WANG; WANG, 2020) & Os autores tem a intenção de aplicar a arquitetura de computador de borda para outras máquinas \\
\hline (OJO et al ., 2021) & Indicam melhorias em seu método para reduzir os falsos positivos. \\
\hline
\end{tabular}

QUADRO 11 - Sugestão de melhorias nos modelos pesquisados. Fonte: Os autores. 
4.3.2 Expansão do atual modelo: os trabalhos do Quadro 12 mostram a intenção dos autores em expandir seus modelos.

\begin{tabular}{|l|l|}
\hline \multicolumn{1}{|c|}{ Trabalhos } & \multicolumn{1}{c|}{ Descrição das oportunidades } \\
\hline (CHEN; LI, 2017) & Indicam o uso do modelo estudo com fusão de dados \\
\hline $\begin{array}{l}\text { (WANG et al. , 2019) \& } \\
\text { (JIANG et al ., 2019) }\end{array}$ & Mencionam a utilização do modelo para atuar sobre dados brutos. \\
\hline (WANG et al. , 2018) & Sugerem expandir a utilização do modelo para sistemas mais complexos. \\
\hline (PENG et al. , 2019) & Pensam em introduzir (TF) e multi-scale learning no atual sistema \\
\hline (EREN, 2017) & Supõe a implementação de hardware com FGPA para o modelo \\
\hline (ZHANG et al. , 2020) & Apontam a utilização de mais funções de ativação e classificadores \\
\hline (WANG et al., 2019) & Introduzem a ideia de aplicar a técnica de multi-kernel para predição \\
\hline
\end{tabular}

QUADRO 12 - Sugestão de expansão dos sistemas propostos. Fonte: Os autores.

4.3.3 Demais oportunidades: demais oportunidades são encontradas no Quadro 13.

\begin{tabular}{|l|l|}
\hline \multicolumn{1}{|c|}{ Trabalhos } & \multicolumn{1}{c|}{ Descrição das oportunidades } \\
\hline (WEN et al. , 2018) & Apontam para o uso de TF com intenção de reduzir o tempo de treinamento \\
\hline (JIANG et al. , 2019) & Irão trabalhar para melhorar o modelo com o incremento de dados desbalanceados \\
\hline (HOANG; KANG, 2019) & Indicam a necessidade de criar um método de seleção automática dos hiper parâmetros \\
\hline (LEI; LIU; JIANG, 2019) & $\begin{array}{l}\text { Menciona a oportunidade de utilizar CNN para reduzir dimensões dos dados de entrada para } \\
\text { métodos LSTM }\end{array}$ \\
\hline (PARK et al. , 2018) & Sugere o uso de modelos mais simples no Edge como GRU para reduzir o processamento \\
\hline (QIAO; WANG; WANG, 2020) & $\begin{array}{l}\text { Aponta para a necessidade de relacionar desgaste de ferramenta com perda de qualidade da } \\
\text { peça }\end{array}$ \\
\hline
\end{tabular}

QUADRO 13 - Oportunidades complementares encontradas na pesquisa. Fonte: Os autores.

\section{CONCLUSÃO}

A revisão sistêmica e bibliométrica realizada nesse trabalho com o uso do método Proknow-C retornou 38 artigos alinhados com o tema de diagnóstico de falhas em equipamentos em tempo real. A análise bibliométrica resgatou as palavras-chaves mais utilizadas, com destaque para "fault diagnosis" e "deep learning”.

Já a análise sistêmica apontou para a redução de custos, a partir da detecção de falhas em equipamentos de forma precoce através de ML. Porém, técnicas estatísticas possuem dificuldades para extrair features de grandes quantidades de dados e sistemas complexos. A solução encontrada foi a aplicação de DL. Essa técnica tem a capacidade de extrair features de forma automática, além de possuir maior facilidade de aprender com múltiplos sensores e dados com diferentes distribuições. 
CNN e LSTM foram as redes neurais mais encontradas na pesquisa. A primeira reduz a dimensão de dados, enquanto a segunda realiza predições com dados em tempo real. Como oportunidade de pesquisa, foi sugerido o uso de TL na intenção de reduzir o tempo de treinamento dos algoritmos. Também, observou a lacuna de algoritmos mais leves para trabalharem em computação de borda e por último a busca pelo uso de EL, a qual se utiliza de diferentes métodos para realizar a classificação de múltiplas falhas de maneira mais precisa.

\section{AGRADECIMENTOS}

Esse trabalho é apoiado pela Fundação de Desenvolvimento da Pesquisa - Fundep - Rota 2030.

\section{REFERÊNCIAS}

ALI, M. Z. et al. Single- and multi-fault diagnosis using machine learning for variable frequency drive-fed induction motors. IEEE Transactions on Industry Applications, v. 56, n. 3, p. 2324-2337, 2020.

CABRERA, D. et al. Bayesian approach and time series dimensionality reduction to LSTMbased model-building for fault diagnosis of a reciprocating compressor. Neurocomputing, v. 380, p. 51-66, 2020.

CHEN, Z.; LI, W. Multisensor feature fusion for bearing fault diagnosis using sparse autoencoder and deep belief network. IEEE Transactions on Instrumentation and Measurement, v. 66, n. 7, p. 1693-1702, 2017.

CHENG, Y. et al. Remaining useful life prognosis based on ensemble long short-term memory neural network. IEEE Transactions on Instrumentation and Measurement, v. 70, Oct. 2021. DOI: 10.1109/TIM.2020.3031113.

ENSSLIN, L. et al. ProKnow-C, knowledge development process - constructivist. [S.1.:s.n.], 2010. Processo técnico com patente de registro pendente junto ao INPI.

EREN, L. Bearing fault detection by one-dimensional convolutional neural networks. Mathematical Problems in Engineering, v. 2017, 2017. DOI: https://doi.org/10.1155/2017/8617315.

EREN, L.; INCE, T.; KIRANYAZ, S. A Generic intelligent bearing fault diagnosis system using compact adaptive 1D CNN classifier. Journal of Signal Processing Systems, v. 91, n. 2, p. 179-189, 2019.

HOANG, D. T.; KANG, H. J. Rolling element bearing fault diagnosis using convolutional neural network and vibration image. Cognitive Systems Research, v. 53, p. 42-50, 2019.

ISLAM, M. M. M.; KIM, J. M. Automated bearing fault diagnosis scheme using 2D representation of wavelet packet transform and deep convolutional neural network. Computers in Industry, v. 106, p. 142-153, 2019.

JI, X. et al. Real-time robust forecasting-aided state estimation of power system based on datadriven models. International Journal of Electrical Power and Energy Systems, v. 125, Aug. 2020. DOI: 10.1016/j.ijepes.2020.106412. 
JIAHAO, Y. et al. SVM-BILSTM: a fault detection method for the gas station IoT system based on deep learning. IEEE Access, v. 8, p. 203712-203723, 2020.

JIANG, G. et al. Multiscale convolutional neural networks for fault diagnosis of wind turbine gearbox. IEEE Transactions on Industrial Electronics, v. 66, n. 4, p. 3196-3207, 2019.

JUN, H. B.; KIRITSIS, D. Several aspects of information flows in PLM. IFIP Advances in Information and Communication Technology, v. 388 AICT, p. 14-24, 2012.

LEE, K. B.; CHEON, S.; KIM, C. O. A Convolutional neural network for fault classification and diagnosis in semiconductor manufacturing processes. IEEE Transactions on Semiconductor Manufacturing, v. 30, n. 2, p. 135-142, 2017.

LEI, J.; LIU, C.; JIANG, D. Fault diagnosis of wind turbine based on Long Short-term memory networks. Renewable Energy, v.133, p.422-432,2019. DOI: 10.1016/j.renene.2018:i:c:p:422432.

LI, X.; ZHANG, W.; DING, Q. A Robust intelligent fault diagnosis method for rolling element bearings based on deep distance metric learning. Neurocomputing, v. 310, p. 77-95, 2018.

OJO, O. et al. A Neural network based method for thermal fault detection in lithium-ion batteries. IEEE Transactions on Industrial Electronics, v. 68, n. 5, p. 4068-4078, 2021.

PARK, D. et al. Lired: a light-weight real-time fault detection system for edge computing using LSTM recurrent neural networks. Sensors (Switzerland), v. 18, n. 7, 2018. DOI: https://doi.org/10.3390/s18072110.

PENG, D. et al. A Novel deeper one-dimensional CNN with residual learning for fault diagnosis of wheelset bearings in high-speed trains. IEEE Access, v. 7, p. 10278-12093, 2019.

QIAO, H.; WANG, T.; WANG, P. A Tool wear monitoring and prediction system based on multiscale deep learning models and fog computing. International Journal of Advanced Manufacturing Technology, v. 108, n. 7-8, p. 2367-2384, 2020.

QU, J. et al. A Neural-network-based method for RUL prediction and SOH monitoring of lithium-ion battery. IEEE Access, v. 7, p. 87178-87191, 2019.

RAZAVI-FAR, R. et al. An Integrated imputation-prediction scheme for prognostics of battery data with missing observations. Expert Systems with Applications, v. 115, p. 709-723, 2019.

SHI, W. et al. Edge computing: vision and challenges. IEEE Internet of Things Journal, v. 3, n. 5, p. 637-646, 2016.

SUN, R. et al. A Coupling diagnosis method of sensors faults in gas turbine control system. Energy, v. 205, 2020. DOI: 10.1016/j.energy.2020.117999.

SYAFRUDIN, M. et al. Performance analysis of IoT-based sensor, big data processing, and machine learning model for real-time monitoring system in automotive manufacturing. Sensors (Switzerland), v. 18, n. 9, 2018. DOI: https://doi.org/10.3390/s18092946.

WANG, H. et al. Advanced fault diagnosis method for nuclear power plant based on convolutional gated recurrent network and enhanced particle swarm optimization. Annals of Nuclear Energy, v. 151, 2021. DOI: 10.1016/j.anucene.2020.107934.

WANG, S. et al. Convolutional neural network-based hidden Markov models for rolling element bearing fault identification. Knowledge-Based Systems, v. 144, p. 65-76, 2018. 
WANG, Y. et al. Remaining useful life prediction of lithium-ion batteries using support vector regression optimized by artificial bee colony. IEEE Transactions on Vehicular Technology, v. 68, n. 10, p. 9543-9553, 2019.

WEN, L. et al. A New convolutional neural network-based data-driven fault diagnosis method. IEEE Transactions on Industrial Electronics, v. 65, n. 7, p. 5990-5998, 2018.

XIA, M. et al. Data-driven prognosis method using hybrid deep recurrent neural network. Applied Soft Computing Journal, v. 93, 2020. DOI: https://doi.org/10.1016/j.asoc.2020.106351.

XU, Y. et al. A Digital-twin-assisted fault diagnosis using deep transfer learning. IEEE Access, v. 7, p. 19990-19999, 2019.

YU, W.; ZHAO, C. Online fault diagnosis for industrial processes with bayesian network-based probabilistic ensemble learning strategy. IEEE Transactions on Automation Science and Engineering, v. 16, n. 4, p. 1922-1932, 2019.

ZHANG, C. et al. Transformer fault diagnosis method using IoT based monitoring system and ensemble machine learning. Future Generation Computer Systems, v. 108, p. 533-545, 2020.

ZHANG, D. et al. A Data-driven design for fault detection of wind turbines using random forests and XGboost. IEEE Access, v. 6, p. 21020-21031, 2018.

ZHANG, R. et al. Transfer learning with neural networks for bearing fault diagnosis in changing working conditions. IEEE Access, v. 5, p. 14347-14357, 2017.

ZHAO, M. et al. Deep residual networks with dynamically weighted wavelet coefficients for fault diagnosis of planetary gearboxes. IEEE Transactions on Industrial Electronics, v. 65, n. 5, p. 4290-4300, 2018. 